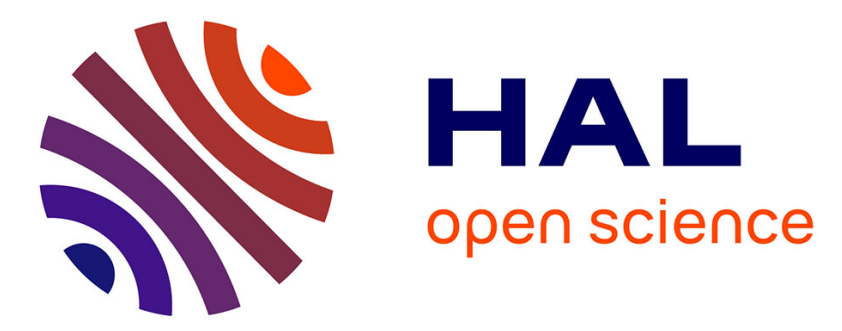

\title{
De Wolfenstein à Half-life : les canons du jeu de combats
}

Olivier Zerbib

\section{To cite this version:}

Olivier Zerbib. De Wolfenstein à Half-life: les canons du jeu de combats : Eléments pour une analyse des formes de création et de réception des jeux vidéo. Protée. Revue internationale de théories et de pratiques sémiotiques, 2002, 30 (1), pp.29-37. 10.7202/006696ar . halshs-00816380

\section{HAL Id: halshs-00816380 \\ https://shs.hal.science/halshs-00816380}

Submitted on 22 Apr 2013

HAL is a multi-disciplinary open access archive for the deposit and dissemination of scientific research documents, whether they are published or not. The documents may come from teaching and research institutions in France or abroad, or from public or private research centers.
L'archive ouverte pluridisciplinaire HAL, est destinée au dépôt et à la diffusion de documents scientifiques de niveau recherche, publiés ou non, émanant des établissements d'enseignement et de recherche français ou étrangers, des laboratoires publics ou privés. 


\section{DE WOLFENSTEIN À HALF-LIFE: LES CANONS DU JEU DE COMBATS ÉLÉMENTS POUR UNE ANALYSE DES FORMES DE CRÉATION ET DE RÉCEPTION DES JEUX VIDÉO}

OLIVIER ZERBiB

Depuis une vingtaine d'années, en passant des arrières salles de bar à l'espace domestique, les productions du secteur du jeu vidéo ont vu leurs formes constamment renouvelées et diversifiées. La multiplication des supports de ces jeux, délaissant en partie l'ordinateur personnel pour les consoles reliées au téléviseur ou leurs homologues de poche, rend impossible tout projet d'analyse du jeu vidéo en tant qu'ensemble homogène, soumis aux mêmes procédés de production ou aux mêmes contraintes de réception. Il semble par ailleurs tout aussi illusoire de chercher à constituer des catégories qui rendraient compte de l'ensemble des titres disponibles sur un marché dont on estime aujourd'hui le chiffre d'affaires mondial à plus de 20 milliards d'euros.

On rencontre cependant, dans les colonnes de la presse spécialisée ${ }^{1}$, l'utilisation récurrente de quelques catégorisations censées mettre un peu d'ordre dans la profusion des jeux proposés au public. On parle ainsi couramment de jeux d'action ou d'arcade pour désigner les productions dans lesquelles le joueur doit faire évoluer un personnage sur l'écran afin d'établir un score ou passer d'un niveau à un autre, de jeux de stratégie lorsqu'il s'agit plutôt de déplacer un ensemble d'unités dans le but de planifier des attaques contre un adversaire virtuel ou humain (dans le cas des jeux en réseau), de simulations pour désigner les jeux se rapportant aux sports ou au pilotage de véhicules en tous genres, et enfin de jeux de rôle ou d'aventure lorsque le joueur est immergé dans une quête au long cours, impliquant un «avatar", sorte de double virtuel, confronté à une série d'énigmes à résoudre. Ces distinctions sont à première vue rassurantes car elles laissent penser que le secteur du jeu vidéo, malgré sa courte histoire, aurait vu se constituer au fil des années quelques genres permettant aux éditeurs de cibler des fractions de public aux attentes nettement différenciées. On imagine ainsi que les jeux dits d'action s'adressent en premier lieu aux plus jeunes du fait de la confrontation presque physique, en tout cas violente, qui oppose le joueur à des adversaires de toutes natures, tandis que les jeux de stratégie, plus réflexifs, concerneraient davantage les adultes. Il convient cependant de s'en méfier, d'abord parce que, comme le signale Jean-Paul Lafrance ${ }^{2}$, on connaît mal les catégories de joueurs à qui s'adressent les jeux, mais surtout parce que l'on assiste fréquemment à 
l'apparition de titres proposant une hybridation entre les genres que nous venons d'évoquer. Ainsi, un jeu tel que Battlezone, créé en 1998 par la société

Activision, est-il décrit par la critique lors de sa sortie sur le marché comme un jeu de stratégie-action, puisqu'il permet au joueur de passer du statut de général, gérant le comportement de toutes les unités de son camp, à celui de simple soldat, sautant d'un véhicule à l'autre pour participer aux combats en «temps réel». Cet exemple n'est évidemment pas isolé et l'on rencontre très tôt dans l'histoire du jeu vidéo des simulations relevant plus du jeu d'arcade que du simulateur de vol, des jeux d'aventures fortement teintés de stratégies, etc.

Difficile donc d'y voir clair dans cette profusion de créations présentées sur le marché à un rythme soutenu. Pourtant, certaines productions américaines, japonaises ou européennes entretiennent les unes avec les autres des relations de ressemblance à ce point frappantes qu'il est possible de supposer que, même pour une pratique culturelle émergente telle que le jeu vidéo, des processus de rationalisation sont à l'œuvre qui orientent le travail des concepteurs selon quelques lignes directrices. S'il ne paraît pas possible d'étendre l'observation de ces processus à des genres bien déterminés, il nous faudra donc en étudier les aspects sur une série limitée de titres, choisis selon leur inscription chronologique dans ce qui nous paraît pouvoir être défini comme une histoire commune.

En la matière, les jeux d'action en trois dimensions présentent un intérêt particulier puisque cette forme de jeux, caractérisée par la mise en scène de combats en vue subjective (le joueur «voit» ses adversaires comme le ferait le héros qu'il commande), a bénéficié d'un grand nombre d'innovations techniques tout en conservant une étonnante stabilité dans les dispositifs narratifs qu'elle propose à ses utilisateurs depuis une dizaine d'années. On se trouve en fait confronté à un paradoxe selon lequel les titres les plus innovants en termes purement techniques, ceux qui, entre tous, réclament pour pouvoir fonctionner les machines les plus performantes, dotées des dernières sophistications informatiques (on pense notamment aux cartes graphiques accélératrices ou encore aux microprocesseurs de dernières générations), sont également ceux qui, nous le verrons, proposent aux joueurs les interfaces ${ }^{3}$ les plus stables dans le temps. En d'autres termes, la série considérable d'innovations techniques que ce secteur du jeu vidéo a connu durant sa courte histoire n'a fait évoluer ni le concept du jeu, qui reste invariablement le même (éliminer un nombre maximum d'ennemis afin de passer d'un niveau à un autre), ni la façon de représenter l'action à l'écran (utilisation de la vue subjective, présence d'indicateurs d'armes et de munitions à l'écran, univers en trois dimensions, etc.). Si la grande majorité des recherches en sciences sociales portant sur les applications des nouvelles technologies fait jouer un rôle central à l'innovation et au progrès technique ${ }^{4}$, il faut convenir que, pour l'objet qui nous importe ici, ce type d'approche s'avère peu heuristique. Il importe moins en effet de retracer l'historique des potentialités techniques mises à disposition des concepteurs de jeux que de comprendre comment ces derniers ont concrètement asservi ces moyens à une intention invariable: plonger le joueur dans un univers en trois dimensions le plus réaliste possible. Pour parvenir à leurs fins, ces créateurs ont certes dû développer des solutions spécifiques à la pratique du jeu vidéo, mais il semble également qu'ils aient importé une partie de leurs modèles du cinéma ou de la littérature, ce qui n'est pas sans conséquence dans la réception de leurs produits par les publics.

\section{LE JEU DE COMBATS}

SOUMIS AUX PRINCIPES DU RÉALISME

Lorsqu'en 1992, la firme ID Software décide de donner une suite à son fameux titre Castle Wolfenstein, elle choisit de tirer profit des derniers progrès du génie logiciel pour rendre plus réaliste la représentation de l'action à l'écran. Si le but du jeu reste identique (s'échapper de la forteresse nazie), l'apparence de cette simulation prend une autre forme avec l'introduction de la troisième dimension. Le titre ainsi développé s'intitulera donc Wolfenstein 3D. L'utilisation d'une vue 
en caméra subjective, qui remplace la vision du personnage de profil se déplaçant dans le plan d'une salle prenant la taille de l'écran, est d'autant plus saisissante que le joueur est amené à se déplacer dans un espace clos, formé d'une longue série de couloirs labyrinthiques. La même équipe développera une succession de jeux reposant sur les mêmes principes (Doom, Doom II, puis la trilogie des Quake), jeux qui deviendront à chaque fois des modèles du genre pour les joueurs ou les firmes concurrentes, à tel point que ces dernières rachèteront parfois la licence du logiciel de rendu en trois dimensions de ces titres pour créer leurs propres jeux ${ }^{5}$. De fait, les concepteurs de Wolfenstein 3D posent les bases d'une interface de jeu qui s'impose aujourd'hui encore comme un standard. L'action du jeu implique que le joueur tienne compte de l'état de ses munitions et de ses blessures pour mener à bien sa mission. Or, la vue en caméra subjective empêche par définition le joueur d'observer son personnage de l'extérieur: il lui est donc impossible de constater la dégradation de l'état de santé de son avatar ou la diminution de son stock d'armes. Les concepteurs ont alors choisi de faire figurer ce type d'informations sous la forme d'indicateurs situés au bas de l'écran vidéo; indicateurs que l'on retrouvera systématiquement dans tous les jeux du même type développés par la suite. De Doom II à Half life, deux jeux produits à quatre années d'intervalle par deux sociétés différentes, on constate ainsi la permanence des solutions adoptées par les concepteurs pour présenter ces informations cruciales pour le joueur ( $c f$. fig. 1 et 2). On notera tout juste une tendance à la simplification de ces indicateurs, simplification qui permet de laisser une plus grande place à la présentation à l'écran de l'action, ce qui est d'ailleurs rendu possible par l'amélioration du rendement des micro-ordinateurs.

Wolfenstein 3D se caractérise enfin par l'utilisation conjointe de la souris et du clavier par le joueur afin de gérer simultanément le maniement des armes et les déplacements du personnage. La plupart des jeux du même style reprendront à leur compte cette innovation, et il est rare aujourd'hui de rencontrer des

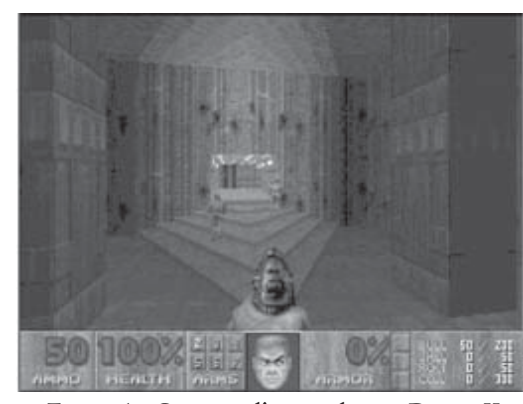

Figure 1 - Capture d'écran du jeu Doom II (ID Software 1995)

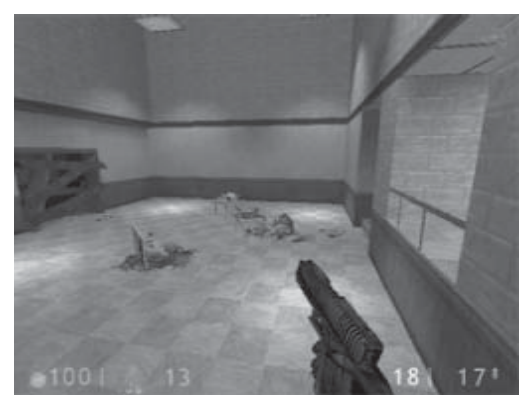

Figure 2 - Capture d'écran du jeu Half-Life (Sierra 1999)

jeux de combats en trois dimensions recommandant l'utilisation du joystick, moins pratique. On voit donc que Wolfenstein 3D est un jeu qui combine subtilement tous les ingrédients du réalisme, tant dans la mise en scène de l'action que dans le maniement du personnage. Bien entendu, ce réalisme est tout relatif, ne serait-ce que parce qu'il est soumis aux limitations qui s'imposent à toute forme de représentation picturale. Comme l'indique Nelson Goodman, «le réalisme est relatif, déterminé par le système de représentation qui sert de norme à une culture ou à une personne donnée à un moment donné» 6 . L'utilisation de la perspective dans ce jeu n'est qu'un exemple du fait que la perception par les spectateurs de l'espace représenté à l'écran repose sur des habitudes de lecture de l'image, habitudes qui s'acquièrent lentement par la fréquentation d'un système de représentation utilisé dans toutes sortes d'œuvres picturales, photographiques ou cinématographiques.

L'univers du jeu vidéo présente cependant quelques particularités en ce domaine, puisque l'évolution des moyens techniques mis à la disposition des concepteurs de jeux a fait lentement varier les conventions utilisées pour dépeindre l'action. On 
passe en effet d'un univers en trois dimensions assez fruste dans Wolfenstein 3D, sans ombres ni textures, à des représentations beaucoup plus proches des images cinématographiques dans des jeux tels que Half-life ou Igi project, dans lesquels les effets de transparence, d'ombrages et de reliefs des objets sont restitués avec beaucoup plus de détails. Les progrès qui ont été franchis entre ces différents jeux ne doivent pas être mesurés en termes purement esthétiques, mais plutôt entendus comme l'expression de ce que Max Weber appelle une rationalité technique progressive 7 des moyens mis en œuvre par les créateurs pour parvenir à leurs fins: immerger le joueur dans un environnement de plus en plus complexe et rendre ainsi l'action plus trépidante. En d'autres termes, la beauté ou le réalisme des décors ne sont pas tant recherchés que le réalisme de l'action dans son ensemble. Ainsi, conserver la fluidité des animations à l'écran est primordial dans un jeu de combats, et tous les titres qui ont mis l'accent sur le réalisme du décor au détriment de cette fluidité, et donc de la vitesse de déplacement du personnage par le joueur, se sont rapidement soldés par des échecs commerciaux cuisants. Inversement, si des titres comme Doom ou Quake se sont imposés comme des modèles au moment de leur sortie sur le marché, c'est uniquement parce qu'ils se présentaient aux joueurs en un compromis réussi entre fluidité de l'action et qualité graphique. Bien entendu, les critères de cette qualité graphique ont progressivement été revus à la hausse en une dizaine d'années avec la mise au point de moteurs graphiques de plus en plus performants: le nombre de détails présents à l'image, le rendu de la lumière et des zones d'ombres, la complexité des effets d'explosions ou de transparence sont quelques-uns des facteurs dont le niveau minimum est conventionnellement fixé par la critique et les joueurs à une époque donnée selon l'état des techniques et de la concurrence. À l'heure actuelle, il est inconcevable d'imaginer un jeu de combats qui ne tire pas profit des cartes accélératrices 3D pour représenter la fumée des explosions ou la brume d'un étang, ce qui restait hors de portée il y a seulement deux ou trois ans. On voit donc que ce qu'il est acceptable aujourd'hui d'envisager comme le standard du réalisme graphique correspond à ce qui, hier, était considéré comme le nec plus ultra en la matière.

Le caractère cumulatif des innovations intervenant dans le rendu graphique de ces jeux ne doit cependant pas faire oublier que ces dernières ne sont développées que dans la mesure où elles servent l'action qui est représentée à l'écran: pouvoir restituer la splendeur d'une lumière automnale ne sert à rien si l'action doit se dérouler entièrement en intérieur... En revanche, la transparence des matériaux est très utilisée car elle permet aux joueurs d'abattre un ennemi placé derrière une vitre, satisfaisant ainsi doublement leur désir de destruction en affectant aussi bien le décor que leur adversaire. Par ailleurs, ce type d'effet permet d'envisager des scénarios de combats plus complexes, le joueur pouvant par exemple être repéré par ses ennemis avant même de pénétrer à l'intérieur d'une pièce. Inversement, les ombres autorisent bien souvent la détection des ennemis placés en hauteur et pourtant situés hors du champ de la caméra subjective.

Le réalisme de l'action se mesure également aux comportements des adversaires: écervelés dans les premiers jeux de combats, ceux-ci ne faisaient rien d'autre que se placer directement dans le champ de vision du joueur en tirant tous azimuts. Peu à peu, les développeurs de jeux ont cherché à supprimer les déplacements erratiques de ces personnages et à augmenter la précision de leurs tirs. Half-life marque un tournant décisif en matière d'intelligence artificielle des opposants en scénarisant à un niveau encore jamais atteint leur comportement de combat: loin de s'offrir en chair à canon pour joueur brutal, ces derniers planifient en groupe leurs attaques et tendent des embuscades; blessés, ils se replient et donnent l'alerte, etc. D'autres équipes de développement ont quant à elles tiré profit de l'idée selon laquelle aucun adversaire n'est plus imprévisible qu'un joueur humain: elles ont donc mis l'accent sur le mode multi-joueurs de leurs titres en privilégiant la fluidité de l'action sur la qualité des décors ou la 
scénarisation des affrontements afin de ne pas allonger outre mesure les temps de chargement des programmes en réseau ou sur Internet.

L'histoire interne de ce type de jeux est donc gouvernée par une série de transpositions, d'un titre à l'autre, d'une interface aux éléments relativement stables, progressivement améliorée dans ses aspects graphiques ou dans la programmation $\mathrm{du}$ comportement des acteurs du jeu, ceci afin d'accroître le réalisme de la simulation. Cette histoire n'est cependant pas continue et certaines évolutions ont parfois conduit à des impasses ou à des bégaiements: le nombre d'armes disponibles, par exemple, passé une période de surenchère, s'est stabilisé aux environs de la dizaine, un arsenal plus important ne permettant pas aux joueurs d'y accéder rapidement (puisque le clavier numérique ne compte que dix touches différentes, de 0 à 9). Le degré de difficulté global des jeux a également été rationalisé de manière à satisfaire différentes catégories de joueurs, des débutants aux experts (fréquemment qualifiés du surnom de hardgamers): on rencontre plutôt aujourd'hui trois niveaux de difficultés (facile, moyen, difficile) au lieu des cinq à sept niveaux des débuts, dont les échelons intermédiaires semblaient peu utiles.

Mais l'uniformisation du format de ces jeux, le fait qu'ils instaurent un dialogue entre les joueurs et leurs machines sous une forme stabilisée ne peuvent être compris uniquement au travers des soubresauts d'une histoire de courte durée qui se limite aux changements intervenus dans certains secteurs de la sphère informatique. Ce serait en effet accorder un trop grand crédit à la nouveauté dans des sociétés où les pratiques culturelles ne se renouvellent que très lentement. Si les concepteurs et les adeptes de jeux vidéo sont un peu trop facilement crédités d'une image d'individus autistes, enfermés dans un rapport fusionnel et quasi exclusif avec leur ordinateur, il faut bien évidemment considérer qu'ils ne vivent pas dans une bulle informatique, coupés du monde, en dehors des influences d'autres activités culturelles. L'imagination créatrice des uns, la disponibilité interprétative des autres sont à coup sûr alimentées par les images et les symboles qui circulent ailleurs, dans les productions issues d'autres formes culturelles.

\section{COMMENT LE CINÉMA D'ACTION, LA LITTÉRATURE} POLICIÈRE ET LA SCIENCE-FICTION FOURNISSENT QUELQUES CARTOUCHES AU JEU DE COMBATS

En prenant un peu de recul, on peut être surpris que les amateurs de jeux de combats admettent l'idée de passer plusieurs heures face à l'écran de leur ordinateur à massacrer un nombre incalculable d'ennemis sur la base d'un scénario souvent très fruste, bref à participer à une action pour le moins irréaliste dans un univers en trois dimensions qui, lui, est censé s'exprimer avec tous les accents du réalisme. On ne peut se satisfaire de l'idée toute faite selon laquelle ces joueurs trouveraient là le moyen d'extérioriser leurs pulsions les plus malsaines, en prenant alors le risque de confondre l'univers virtuel qui leur est proposé avec la réalité: les limites des thèses condamnant les dangers de la violence au cinéma, à la télévision ou dans les jeux vidéo ayant été souvent soulignées dans de nombreux articles, il n'est sans doute pas utile d'y revenir ici. Disons simplement, pour reprendre les propos du psychanalyste D.W. Winnicott ${ }^{8}$, que le jeu constitue un espace imaginaire au sein duquel les activités symboliques des individus permettent de réorganiser la réalité, ce qui s'avère être sans conséquence sur la vie ordinaire de ces derniers. Et, bien que reposant sur des dispositifs multimédias, les jeux de combats n'ont pas cette faculté que l'on prête un peu naïvement à l'audiovisuel ${ }^{9}$ de rendre plus immédiate et prégnante la communication entre l'utilisateur et sa machine, de réduire la fracture entre la réalité et ses représentations. Non, les joueurs ne doivent sans doute pas leur propension à accepter un concept de jeu aussi violent aux particularités de la médiation informatique, mais plutôt, pensons-nous, aux nombreuses références cinématographiques ou littéraires qui proposent des univers narratifs assez semblables à ceux qui sont restitués dans les titres de jeu de combats. De James Bond à Rambo, en effet, il est possible d'énumérer un grand nombre de films 
opposant un héros invincible (ou en tous cas peu sujet aux blessures) à une cohorte d'ennemis dépersonnalisés, substituables les uns aux autres. La figure emblématique du "méchant», ennemi particulièrement pervers et coriace souvent représenté dans ces fictions, est d'ailleurs parfois présent dans les jeux de combats sous la forme du «big boss», adversaire mieux armé et plus résistant que les autres, dont l'élimination ouvre les portes du niveau de jeu suivant.

Ces films ou romans ne constituent le plus souvent qu'une source d'inspiration pour les créateurs de jeux, les adaptations fidèles demeurant relativement rares. Les romans d'H.P Lovecraft, par exemple, n’ont pas réellement été adaptés dans les jeux vidéos, mais ont vraisemblablement inspiré à de nombreux titres leur ambiance angoissante et glauque et leur univers peuplé de mutants et de créatures semidivines. En fait, la plupart des titres qui mettent directement en scène un héros tiré du cinéma d'action ou de la littérature policière et de science-fiction se sont peu vendus, sans doute parce que l'achat coûteux des licences d'exploitation en a grevé le budget de développement: les jeux ainsi créés innovent peu ou pas du tout en matière de programmation, ce qui s'avère être un handicap considérable dans un secteur où les progrès, on l'a dit, s'opèrent très rapidement. On notera tout de même l'exception notable des jeux produits par Tom Clancy, auteur américain à succès de romans d'espionnage, qui a participé en personne, semble-t-il, à la mise sur le marché de la série des Rainbow Six.

Les emprunts au cinéma se révèlent encore plus directement, dans la grande majorité des jeux de combats, par la présence de scènes dites "cinématiques", servant à introduire l'action ou à établir la transition entre différentes phases du jeu. Ces scènes entièrement numérisées, que le joueur peut interrompre à tout moment mais sur le déroulement desquelles il n'a aucune prise, prennent la forme de courts films d'animation, comme on peut en voir dans des salons de création numérique tels qu'Imagina. Les procédés diégétiques utilisés sont directement importés du cinéma et, de fait, ces cinématiques ne diffèrent guère de ce que les films d'action donnent à voir à leurs spectateurs durant leurs premières minutes. C'est ainsi l'occasion pour le joueur de prendre connaissance des ressorts principaux de l'action et de comprendre le rôle que son personnage doit y tenir. L'existence de telles séquences d'animation, si elle se justifie grandement par le besoin qu'ont les concepteurs de ne pas immerger le joueur dans l'action avant d'en avoir planté le décor, est également grandement motivée par la volonté qu'ont ces mêmes concepteurs de faire montre de leur virtuosité technique en réalisant les cinématiques les plus spectaculaires possible. Les images numériques qui les composent sont en effet à ce point travaillées que l'on observe un écart considérable entre le niveau graphique atteint dans ces séquences et celui qui constitue l'univers du jeu à proprement parler. Tout se passe ici comme si la bande-annonce d'un film était réalisée en cinémascope alors que le film lui-même l'était en format vidéo. L'effet obtenu est à ce point frappant que la critique a pris pour habitude d'évaluer séparément le rendu graphique des cinématiques et celui des séquences de jeu. Même si les techniques actuelles de programmation permettent de réduire ces écarts en améliorant l'apparence des décors, des personnages et des éléments de jeu, on voit que l'on a affaire ici à une part non (encore) rationalisée des procédés de narration utilisés dans les jeux vidéo, ou à tout le moins à des éléments qui tirent leur rationalité d'univers de création différents et dont la fusion est encore imparfaite.

L'ambiance sonore et musicale, très couramment utilisée dans les jeux de combats pour susciter l'angoisse et le suspens chez les joueurs, s'inspire quant à elle du cinéma. Passés les premiers temps de l'histoire du jeu vidéo dans lesquels les bruitages et les musiques demeuraient simplistes, les concepteurs de jeux prennent actuellement grand soin des éléments sonores qu'ils intègrent à leurs productions. L'utilisation de la stéréophonie, d'effets spéciaux, d'enregistrements de bruits réels (comme le tir d'une mitrailleuse, le son des balles entrant dans le chargeur 
d'un revolver, etc.) est devenue monnaie courante, tandis que des musiques différentes sont

fréquemment composées pour chaque séquence de jeu en fonction de l'ambiance recherchée. Lorsque l'on prend connaissance du nom des personnes qui composent l'équipe de développement de tels jeux au travers des "crédits", sorte de générique de fin (lui aussi inspiré du cinéma ou de la télévision), on peut d'ailleurs constater que des groupes spécifiques ont travaillé à la réalisation des effets sonores et des musiques, témoignant ainsi de l'importance nouvelle que revêt la constitution d'une bande sonore complexe pour les éditeurs de jeux vidéo.

Les liens privilégiés qui semblent unir ces deux champs de création distincts que sont le cinéma et le secteur du jeu vidéo apparaissent également dans le mode de structuration professionnelle de ce dernier. Depuis quelques années, en effet, on observe la constitution d'équipes de développement de jeux sur un modèle assez voisin de ce qui existe dans le milieu du cinéma, au tout au moins dans la production de films à petits ou moyens budgets. À mesure que le marché du jeu vidéo se professionnalise, laissant moins de place aux autodidactes de génie pour privilégier la production de titres aux aspects marketing soigneusement étudiés ${ }^{10}$ (la création d'un jeu coûte actuellement plusieurs millions d'euros, dont près de la moitié est consacrée au marketing et au lancement sur le marché), de nouveaux métiers apparaissent qui font inévitablement penser à ceux que l'on trouve au cinéma: producteur, producteur exécutif, directeur artistique, directeur de casting (pour l'enregistrement de la voix des personnages), etc. Par ailleurs, les équipes de développeurs se constituent en pôles d'animation assez semblables à ceux qui existent dans les studios d'animation tels que Disney ou Dreamworks, studios qui éditent d'ailleurs de nombreux jeux vidéo. Les frontières entre jeu vidéo et cinéma sont donc loin d'être étanches, surtout lorsque l'on sait que les créateurs d'effets spéciaux, très sollicités par les studios hollywoodiens ou européens, négocient régulièrement leurs services auprès des équipes de développement de jeux vidéo.
On saisit mieux dès lors que la standardisation progressive des jeux de combats, au fil des années, répond en partie à l'évolution opérée par le secteur du jeu vidéo dans son ensemble vers un modèle de production proche de celui qui régit l'économie mondiale du cinéma. La place laissée aux créateurs dans ce modèle étant quelque peu amoindrie en regard de ce qu'elle pouvait être au temps des pionniers de la micro-informatique, on ne s'étonnera pas du fait que les jeux de combats qui paraissent actuellement sur le marché semblent, plus qu'hier, calibrés sur un standard dont les éléments ne varient guère. Pour autant, et même pour une activité en apparence aussi fruste que celle qui consiste à éliminer le plus grand nombre possible d'adversaires, il n'est pas possible de réduire l'activité interprétative des publics de ces divertissements informatiques à l'univocité de leur concept de jeu.

\section{MATÉRIAUX POUR UNE ANALYSE}

NON EXCLUSIVEMENT «MATÉRIELLE»

DE LA RÉCEPTION DES JEUX VIDÉO

Le plus souvent, lorsque l'on cherche à décrire l'activité interprétative des joueurs face à leur machine, on en vient à analyser leurs relations psychologiques ou psychosociologiques au jeu ou à étudier leurs rapports à la technique informatique et aux valeurs qui y sont rattachées. En montrant que les jeux de combats entretiennent avec d'autres formes culturelles des contacts troublants, il n'est pas question pour nous d'affirmer que l'on joue à ces jeux comme on lit un roman policier ou comme on regarde un film d'action, mais plutôt de souligner combien ces références culturelles servent de cadre à la réception de ces jeux par leurs publics, un cadre qui dépasse donc nettement les frontières de la médiation informatique. Patricia M. Greenfield ${ }^{11}$, dans un article consacré à la diffusion sociale de procédés de perception et de connaissance par le biais des jeux vidéo, a démontré comment la perception de l'espace représenté à l'écran par les joueurs était soutenue par leurs habitudes télévisuelles et cinématographiques. Ce faisant, elle nous prouve combien il est important 
de ne pas cloisonner l'étude du comportement des joueurs à la simple relation technique qui les unit à l'ordinateur.

Ainsi, on explique couramment l'apparente surreprésentation des hommes dans les amateurs de jeux vidéo, et singulièrement de jeux de combats, par le fait que les femmes se montreraient moins intéressées par la technique, et donc l'informatique, que leurs homologues masculins, réservant alors leurs loisirs à d'autres pratiques culturelles. En accordant autant d'importance aux aspects techniques de la relation qui s'engage entre un usager de l'informatique et son ordinateur, on simplifie sans doute un peu les choses et l'on oublie à quel point la domestication d'un outil technique, quel qu'il soit, repose moins sur l'apprentissage de son fonctionnement que sur les logiques d'usages qu'un individu peut développer à son égard. En d'autres termes, et sans chercher à simplifier outre mesure, disons que si les femmes s'intéressent moins à l'informatique que les hommes, peut-être est-ce tout simplement que les applications qui leur sont proposées, ici les jeux vidéo, les intéressent peu ou pas du tout! On sait en effet que les hommes cèdent plus facilement aux plaisirs d'un roman policier ou d'un film d'action, comment s'étonner dès lors qu'ils tirent un plus grand plaisir que les femmes à jouer à un jeu de combats qui multiplie les références à ces formes culturelles? La différence féminine qui s'exprime au travers des usages ludiques de la micro-informatique s'observe, on le sait, pour de nombreuses autres pratiques culturelles, et l'on aurait tort de faire jouer aux médiations matérielles un rôle central, surtout lorsque l'on veut bien considérer que, sur d'autres terrains, à commencer par l'espace domestique, les femmes sont encore amenées à manipuler davantage d'objets techniques que les hommes; preuve s'il en était besoin qu'elles ne montrent pas systématiquement une défiance à l'égard des innovations technologiques. L'étude de la réception des jeux vidéo ne peut donc pas à nos yeux être cantonnée à l'analyse des pratiques informatiques des publics de ces jeux (ce qui reviendrait à commenter platement des niveaux de compétences techniques, comme on le fait insidieusement pour expliquer les différences de sexes), mais doit également tenir compte des formes cinématographiques et littéraires dont la rencontre détermine pour une part importante l'interprétation qui pourra être faite d'un titre singulier.

Les méthodes d'analyse développées en sociologie de la réception des œuvres littéraires ou filmiques nous paraissent donc heuristiques pour comprendre comment, malgré leur standardisation, les jeux de combats peuvent être domestiqués fort différentiellement selon le cadre culturel qui régit leur interprétation. Il en est ainsi, par exemple, des travaux menés par E. Ethis sur la symbolique temporelle au cinéma ${ }^{12}$, qui éclairent de façon frappante combien la différence entre le temps vécu par les spectateurs lors de la projection d'un film et celui qu'ils auront effectivement perçu signale des modes de réception hétérogènes. On ne peut être que frappé de la difficulté qu'ont la plupart des amateurs de jeux vidéo à évaluer précisément le temps qu'ils passent à jouer devant leur écran d'ordinateur, difficulté qui constitue souvent une source de conflits avec l'entourage de ces joueurs (parents ou conjoint). Cette fuite du temps, si elle souligne le caractère trompeur de la dénomination «en temps réel» qui qualifie régulièrement les jeux de combats, signale surtout les effets de la scénarisation de l'action de ces jeux sur un modèle importé de la diégèse filmique et ouvre un champ d'investigation fort intéressant pour la recherche. Il ne suffit pas en effet de constater des inégalités d'appréciation temporelle chez les joueurs, il s'agira encore d'en comprendre les ressorts (origine sociale, âge, sexe, niveau de diplôme pour ne parler que des indicateurs classiques de la sociologie).

Dans le même ordre d'idées, il faudrait tenir compte de la référentialité spatiale ou historique inscrite dans le scénario de ces jeux pour en mesurer les différences de réception. En effet, bien qu'un grand nombre de jeux de combats proposent à leurs utilisateurs une immersion dans un univers de science-fiction, il n'est pas rare que l'action se déroule dans une ville ou à une époque précises. Duke Nukem 
3D, par exemple, situe son action dans un New York apocalyptique, peuplé de mutants en tous genres: selon leur nationalité, les joueurs peuvent voir dans ce cadre un détournement de leur quotidien ou bien au contraire une forme d'exotisme un peu particulière... On est loin en tout cas de pouvoir affirmer que la standardisation de ces jeux conduit à l'uniformisation des réceptions qui risquent d'en être faites.

Un grand nombre de pistes de recherches restent donc ouvertes aux chercheurs qui voudraient se pencher sur de tels objets. L'intérêt de ce type d'études ne reposent pas, selon nous, sur le caractère novateur ou récent de ces productions culturelles, mais bien au contraire sur la possibilité qu'elles offrent de faire sortir l'analyse des créations technologiques du débat sur l'innovation à partir duquel elles restent trop souvent abordées. Marc Bloch et Lucien Febvre appelaient déjà en 1935, dans le premier numéro thématique des Annales, à étudier les productions techniques comme des ensembles culturels, en dépassant la vision téléologique de l'histoire dans laquelle les approches technicistes sont condamnées à s'enfermer.

En nous défiant des catégories toutes faites, en ne cherchant pas à réifier les jeux vidéo en un objet de pratiques circonscrites et stables, notre propos n'est pas de dissoudre la spécificité de ces formes de créations contemporaines dans un tout culturel qui serait amené à se reproduire à l'identique indéfiniment. Disons tout au moins que plutôt que tenter de faire dire à ces objets ce que pourra être notre futur, il nous paraît beaucoup plus pertinent de chercher à travers eux l'expression d'une histoire culturelle qui s'exprime de façon renouvelée.

\section{Notes}

1. On pense notamment au Monde Interactif ou à des magazines tels que Joystick, PC Loisirs ou encore Gen 4.

2. J-P. Lafrance, La Machine métaphysique. Matériaux pour une analyse des comportements des Nintendo Kids, Réseaux, nº67, Paris, CNET, 1994.

3. On désigne par ce terme les moyens mis en œuvre par les concepteurs pour organiser les échanges d'informations entre l'utilisateur et le système informatique. La «jouabilité» d'un titre est alors notamment définie par la capacité qu'ont ces concepteurs à synthétiser la présentation à l'écran des informations nécessaires au joueur pour manier son personnage le plus efficacement possible. 4. Voir à ce propos l'article de D. Edgerton, «De l'innovation aux usages. Dix thèses éclectiques sur l'histoire des techniques ", dans Des Sciences et des Techniques: un débat, Paris, Éd. de l'École des Hautes Études en sciences sociales, 1998.

5. On rencontre d'ailleurs souvent dans la presse spécialisée l'utilisation des termes «doom-like» ou "quake-like" pour décrire les jeux de combats en trois dimensions en vue subjective.

6. N. Goodman, Langages de l'art, Nîmes, Éd. Jacqueline Chambon, 1990, p. 62.

7. M. Weber, Essais sur la théorie des sciences, Paris, Plon, 1965.

8. D. W. Winnicott, Jeu et Réalité: l'espace potentiel, Paris, Gallimard, 1975.

9. Voir à ce propos J-C. Passeron, «Les yeux et les oreilles: à propos de l'audiovisuel ", dans Le Raisonnement sociologique, Paris, Nathan, 1991. 10. Voir à ce sujet l'enquête du Monde Interactif du 14 janvier 2001, Jeux vidéo: mais où sont les créateurs français?

11. P.M. Greenfield, «Les Jeux vidéo comme instruments de socialisation cognitive ", Réseaux, n 67, Paris, CNET, 1994.

12. E. Ethis, «Le cinéma et l'empreinte du temps. L'œuvre cinématographique au crible de la réception spectatorielle", Protée, vol. $27, \mathrm{n}^{\circ} 2,1999$. 